\title{
Les femmes dans la filière lait périurbaine au Sénégal. Cas de la région de Kolda
}

\author{
M. Ba Diao ${ }^{1 *}$ C.D. Senghor ${ }^{2}$ B. Diao ${ }^{3}$
}

\section{Mots-clés}

Rôle des femmes - Production laitière - Participation de la femme Politique de l'éducation - Association d'agriculteurs - Sénégal.

\begin{abstract}
Résumé
Une enquête participative a été réalisée en l'an 2000 dans la région de Kolda, en zone soudanienne au sud du Sénégal, pour analyser les freins au développement de la filière lait et déterminer le rôle et l'apport des femmes dans cette filière. Les femmes sont souvent juridiquement propriétaires des bovins, font la traite et assurent la transformation et la commercialisation du lait. Cependant, elles n'ont aucun contrôle sur les facteurs de production, l'accès à l'instruction et au crédit leur est difficile, et leur niveau de participation aux organisations paysannes est faible. L'amélioration de leur situation passe nécessairement par la mise en place d'un programme d'appui comprenant des actions de formation et d'alphabétisation fonctionnelle, ainsi que des mesures d'accompagnement, comme la mise à disposition de matériels allégeant leurs travaux domestiques et la construction $d^{\prime}$ infrastructures physiques de base.
\end{abstract}

\section{INTRODUCTION}

Ces deux dernières décennies, les débats sur les rôles des sexes dans le développement économique et social, et sur l'égalité entre hommes et femmes ont pris une place croissante dans les préoccupations des décideurs et des professionnels du développement. Ils ont surtout permis de noter combien l'appartenance à un sexe donné conditionne l'accès aux ressources matérielles, économiques, la relation au pouvoir, la capacité d'initiative et d'action (19). Mais si une prise de conscience des problèmes est perceptible dans le monde entier, on est encore loin de disposer d'assez de données détaillées sur le travail et la contribution économique des femmes. Cette lacune est particulièrement manifeste en ce qui concerne le rôle des femmes dans le développement agricole et rural (1).

Pourtant en Afrique, les femmes produiraient 80 p. 100 des denrées alimentaires, assumeraient 70 p. 100 du travail agricole, la

\footnotetext{
1. Isra, laboratoire national de l'élevage et de recherches vétérinaires, BP 3120, Dakar, Sénégal

2. Cabinet Performances Management Consulting, BP 22 352, Dakar Ponty, Sénégal

3. Sud-Vetagro, BP 139, Kolda, Sénégal

* Auteur pour la correspondance

Tél. : 2218323678 ; fax 2219322118 -E-mail : mbadiao@sentoo.sn
}

totalité de la transformation des produits de base et 60 à 90 p. 100 de la commercialisation (13). Il est donc impératif aujourd'hui de prendre en compte les problèmes des femmes dans la formulation des politiques de développement agricole. Car, pour mettre en œuvre des interventions qui contribuent véritablement à un développement durable, il faut clairement identifier les producteurs et mieux cerner leurs besoins et leurs priorités.

Ceci pourrait particulièrement être appliqué au cas de la filière laitière dans la région de Kolda au Sénégal. Vouloir développer une filière suppose de bien identifier les acteurs, de déterminer leurs rôles respectifs, d'analyser la dynamique des décisions à tous les niveaux, d'examiner la place faite aux femmes dans les organisations paysannes et surtout leurs besoins et priorités.

L'analyse qui suit résulte d'enquêtes effectuées dans le cadre d'une étude globale sur la filière laitière de la région de Kolda. Son objectif est de répondre à une série de questions : quel est le mode de participation des femmes et des hommes aux activités ? Qui assure les différentes tâches identifiées au sein de la filière laitière ? Qui prend les décisions ? Sur quelles ressources les femmes et les hommes exercent-ils un contrôle ? Quel est le niveau de formation des femmes et quelle est leur possibilité d'accès au crédit ? Quelles stratégies mettre en œuvre pour que les femmes participent de manière plus active au développement de la filière ? 


\section{MATERIEL ET METHODES}

\section{Contexte de l'étude}

L'élevage dans la région de Kolda (sud du Sénégal) est intégré à l'agriculture dans un système de production agropastoral de type traditionnel, caractérisé par l'exploitation de races trypanotolérantes à faible potentiel génétique et à faibles performances de production et de reproduction. C'est dans ce contexte que, depuis une dizaine d'années, la Sodefitex ${ }^{1}$ et le $\mathrm{Crz}^{2}$ de l'Isra ${ }^{3}$ ont mis en place des étables fumières, avec pour objectif l'amélioration de la capacité de travail des animaux de trait et la production de fumier de qualité pour la fertilisation organique des sols. La stabulation des vaches lactantes a permis de produire du lait en saison sèche destiné à l'autoconsommation et à la commercialisation autour des villes de Kolda et de Vélingara. L'augmentation des quantités de lait mises sur le marché a favorisé l'émergence d'unités artisanales de transformation de lait dans ces deux localités (9).

Dans le département de Sédhiou, le Primoca ${ }^{4}$ est intervenu, en matière d'élevage, dans l'organisation des producteurs, la création de mares artificielles, la lutte contre les feux de brousses. Ces actions ont rendu possible le développement d'une production laitière locale. Une unité semi-moderne de fabrication de fromages a été implantée, suite à l'augmentation de l'offre en lait frais.

La filière laitière émergente de la région de Kolda rencontre pourtant un certain nombre de problèmes qui concernent la production, la transformation et la commercialisation. Cette étude, qui s'inscrit dans le cadre de la planification d'un programme d'appui au développement des petites et moyennes entreprises autour de la filière laitière (20), devait permettre de faire le diagnostic de cette filière et de dégager des perspectives de développement durable ainsi que des recommandations pertinentes.

\section{Méthodes}

Une approche participative (8) a été appliquée pour réaliser un état des lieux des différents maillons de la filière lait dans la région de Kolda et identifier les principales contraintes. La zone de l'étude a été circonscrite dans un rayon de $15-25 \mathrm{~km}$ autour des villes de Kolda, Sédhiou et Vélingara. Au-delà de cette distance, l'approvisionnement des villes en lait a été aléatoire compte tenu de l'état des infrastructures routières et de l'absence d'une chaîne de froid. Près d'une centaine de personnes ont été rencontrées, soit individuellement, soit en groupes. Il s'agissait de producteurs, de transformateurs, de vendeurs de lait, mais également de représentants des structures d'encadrement, de recherche, de crédit présentes dans la région.

L'équipe pluridisciplinaire (un zootechnicien, un vétérinaire, un économiste, un nutritionniste et un agro-industriel) a entrepris le travail d'entretiens participatifs pendant trois mois avec des visites répétées sur le terrain et des synthèses continues. Les guides d'entretiens semi-directifs utilisés n'ont pas entravé la libre expression des interviewés, mais ont plutôt évité les digressions. Cette démarche a permis d'effectuer une revue des contraintes socioéconomiques et technologiques, ainsi que d'identifier les opportunités de développement de la filière : types d'exploitation, organisation de la production et de la transformation, niveau d'activités des unités de traitement du lait... L'implication des femmes et des jeunes, les contraintes de formation, les besoins de crédit et d'organisation des acteurs ont pu être analysés. Les résultats présentés ici concernent surtout le rôle des femmes (essentiellement d'ethnie Peule) dans la filière.

\footnotetext{
${ }^{1}$ Société de développement des fibres textiles

${ }^{2}$ Centre de recherches zootechniques

${ }^{3}$ Institut sénégalais de recherches agricoles

${ }^{4}$ Projet intégré pour la Moyenne Casamance
}

\section{RESULTATS}

\section{Présentation générale de la filière}

La filière lait dans la région de Kolda est décrite à la figure 1. Le lait sert, pour une part, à l'alimentation quotidienne de la famille, pour l'autre, à la vente en petites quantités sur marché hebdomadaire ou en ville. Il est vendu aux consommateurs directement par de petits producteurs ainsi que par un ou deux intermédiaires. Une grande partie de la production locale emprunte le système traditionnel de transformation ; toutes les opérations et les manipulations du lait se font manuellement. Les acteurs sont des femmes d'éleveurs et des transformatrices résidant en ville. Les produits sont le lait caillé et l'huile de beurre.

Sept unités de pasteurisation sont implantées dans la région. L'une d'entre elles est spécialisée dans la fabrication de fromages italiens. Elle produit une douzaine de variétés dont les deux plus importantes sont la mozzarella et l'italico (fromage salé à pâte pressée). Du lait caillé aromatisé sucré ou non sucré, conditionné dans des sachets en plastique d'un demi-litre ou d'un quart de litre, est produit par les autres unités.

\section{Place et rôle de la femme au niveau familial}

\section{Participation des femmes dans la filière laitière}

$\mathrm{Au}$ sein de la filière lait à Kolda, la division sexuelle du travail est relativement nette (tableau I). A l'homme reviennent le gardiennage, la conduite au pâturage, l'abreuvement, les soins aux animaux, le transport et l'échange des animaux sur pied. Il peut assumer ces opérations seul ou avec l'assistance de ses fils ou neveux ; il peut aussi louer les services d'un berger.

L'enfant apporte très tôt sa contribution au système. Dès l'âge de 5 ans, sous la surveillance de sa mère, il commence à s'occuper

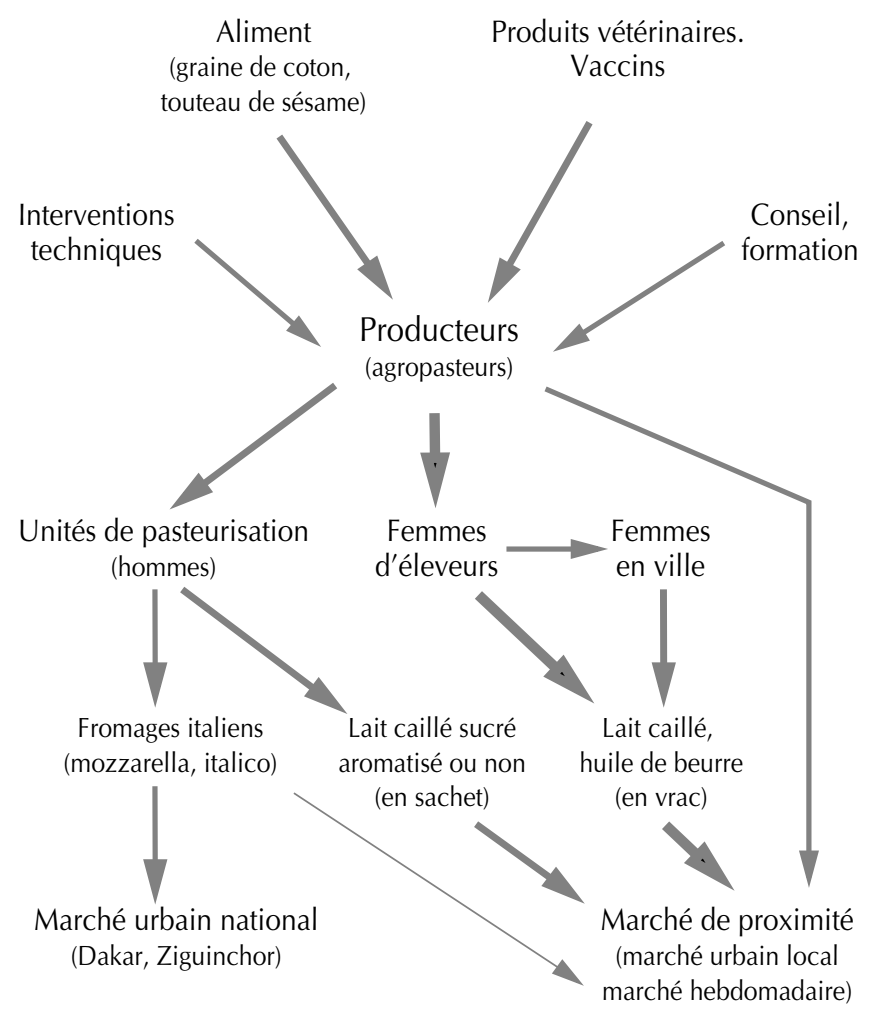

Figure 1 : schéma simplifié de la filière lait dans les zones périurbaines de Kolda. 
des jeunes encore au pis. Il devient à 10-11 ans un jeune berger pour les veaux sevrés et les petits ruminants et il peut suivre les gros troupeaux bovins dès 13 ans. L'abreuvement des animaux en stabulation, les soins aux animaux malades et aux veaux, la traite, la transformation artisanale (en lait caillé et huile de beurre) et la commercialisation des produits sont les tâches traditionnellement dévolues aux femmes. Les auteurs ont cependant noté l'implication de plus en plus importante des hommes dans la transformation (unités de pasteurisation) et dans la commercialisation du lait (vente directe par les producteurs).

\section{Appartenance du capital animal, pouvoir de décision et revenus}

Le tableau II montre que près des deux tiers des bovins appartiennent aux femmes. Elles reçoivent de leur mari (dot) et de leurs parents (héritage), au moment de leur mariage, une ou plusieurs vaches destinée(s) à l'entretien et à l'installation future des enfants. C'est à leur mari qu'incombe la gestion du troupeau. Théoriquement, les hommes ne peuvent vendre des vaches appartenant à leurs femmes qu'avec leur accord préalable. En réalité, ils considèrent bien souvent que le fait de s'occuper du bétail leur donne implicitement ce droit. Cependant, les femmes peuvent faire intervenir des parents pour empêcher la vente du bétail illicite selon la tradition, c'est-à-dire pour d'autres motifs que les cérémonies familiales, les impôts, les pèlerinages. Aucune femme ne peut décider de vendre ses animaux bovins sans l'accord préalable du mari et les transactions sont du ressort exclusif de ce dernier. De ce fait, le mari peut utiliser cet argent sans en référer à son épouse.

La majorité des animaux appartiennent aux femmes, pourtant elles reçoivent une part des produits et des transactions plus faible que les hommes (tableau II) et elles ne peuvent pas disposer entièrement de cette somme pour leurs propres besoins. En effet, les revenus tirés de la vente du lait sont en général destinés à l'achat de produits alimentaires pour la famille, mais aussi d'habits pour les enfants. Cette phrase d'une femme du département de Kolda a bien résumé la situation « lorsque la commercialisation du lait marche bien, les hommes ne se préoccupent plus de la nourriture de la famille; cela nous incombe, à nous les femmes ».

Jusqu'à présent, les femmes ont rarement disposé d'épargnes monétaires issues du troupeau bovin. Cependant, certaines d'entre elles sont arrivées à conserver et faire croître un petit élevage (volaille, petits ruminants) qu'elles ont acquis souvent par don des parents. L'utilisation de ce cheptel par les femmes est plus libre. En effet, elles peuvent abattre, offrir ou vendre (surtout la volaille) sans autorisation préalable. Par ailleurs, des femmes d'origine rurale et installées en ville peuvent faire de la transformation et de la commercialisation du lait un métier leur rapportant plus que le salaire minimum garanti (Smig) en vigueur au Sénégal (tableau III).

\section{Niveau de formation technique des femmes et appui}

Dans le domaine laitier, rares sont les femmes ayant bénéficié de formations. Absentes des séances de formation sur les techniques de production (pratiques de stabulation), quelques-unes d'entre elles (une vingtaine), membres du Directoire des femmes en élevage, ont suivi en 1998 des modules sur l'hygiène et les techniques de transformation artisanale du lait (pasteurisation, ajout de ferments...). Cette formation s'est adressée aux femmes qui vivaient en ville et vendaient déjà du lait caillé ou souhaitaient en vendre. Cependant, ces formations n'ont pas produit les effets escomptés. Seules deux femmes (une à Kolda et une à Sédhiou) transformaient et commercialisaient du lait en l'an 2000.

\section{Niveau de l'appui financier}

Des programmes de financement sur l'élevage étaient disponibles dans le cadre du système financier décentralisé. Toutefois, ils ne

\section{Tableau I}

Implication des membres de la famille dans les activités laitières *

\begin{tabular}{lccc} 
Activités & Hommes & Femmes & Enfants \\
\hline $\begin{array}{l}\text { Conduite au pâturage } \\
\quad \begin{array}{c}\text { Période de cultures** } \\
\quad \text { (juillet-décembre) }\end{array}\end{array}$ & 65 & - & 35 \\
$\quad \begin{array}{l}\text { Période après récolte } \\
\quad \text { janvier-juin) }\end{array}$ & 30 & - & 70 \\
$\quad$ Abreuvement & 50 & 5 & 45 \\
$\begin{array}{l}\text { Soins aux jeunes animaux } \\
\text { et aux malades }\end{array}$ & 20 & 50 & 30 \\
$\begin{array}{l}\text { Traite } \\
\text { Transformation }\end{array}$ & - & 100 & - \\
$\begin{array}{l}\text { Vente de produits laitiers } \\
\text { Vente d'animaux }\end{array}$ & - & 100 & - \\
\end{tabular}

* Etude de cas de 15 familles du village de Saré Guiro Yéro Bocar, département de Kolda

** Certaines familles aisées utilisent les services d'un berger payé 1250 Fcfa par animal pour la période

\section{Tableau II}

Appartenance des animaux et partage des revenus entre hommes et femmes par troupeau *

\begin{tabular}{|c|c|c|c|}
\hline & Total & $\begin{array}{l}\text { Part des } \\
\text { hommes } \\
\quad(\%)\end{array}$ & $\begin{array}{c}\text { Part des } \\
\text { femmes } \\
(\%)\end{array}$ \\
\hline $\begin{array}{l}\text { Nombre d'animaux } \\
\text { par troupeau }\end{array}$ & $87 \pm 23$ & 34,7 & 65,3 \\
\hline \multicolumn{4}{|l|}{$\begin{array}{l}\text { Vente de lait et } \\
\text { d'huile de beurre (Fcfa) }\end{array}$} \\
\hline Mars-juin & 0 & - & - \\
\hline Juillet-novembre & 568125 & 0 & 100 \\
\hline Décembre-février & 114375 & 0 & 100 \\
\hline Total & 682500 & 0 & 100 \\
\hline Vente d'animaux (Fcfa) & 387560 & 76,6 & 23,4 \\
\hline $\begin{array}{l}\text { Revenu annuel moyen } \\
\text { par femme } \\
\text { ou par homme }{ }^{* *}(\mathrm{Fcfa})\end{array}$ & - & 151200 & 108450 \\
\hline
\end{tabular}

* Etude de cas de 15 familles du village de Saré Guiro Yéro Bocar, département de Kolda

** Les familles sont polygames, chaque femme gère le lait de ses vaches et de celles de ses enfants non mariés. Le lait des animaux du mari est récupéré par la femme qui est de tour de cuisine. En moyenne, il y a deux hommes chefs de ménages et sept femmes pour un troupeau.

concernaient que des activités à cycles courts : embouche ovine, production de poulets de chair. Certains groupements de femmes en ville en ont bénéficié. Mais l'absence de dispositifs financiers adaptés à la production laitière demeurait l'une des contraintes majeures freinant le développement de la filière lait. 


\section{Les femmes et les associations de producteurs}

Les organisations paysannes, présentes dans la région de Kolda (tableau IV), sont caractérisées par une grande diversité dans leur forme juridique, leur origine, leurs activités et leur champ d'action, mais elles ont toutes été suscitées de l'extérieur. Elles ont bénéficié de plusieurs programmes et interventions d'organisations non-gouvernementales, d'organismes de coopération et de structures publiques visant à développer la production laitière de la région.
Elles ont eu beaucoup de difficultés à affirmer leur autonomie par rapport à « leurs tutelles »

A ce jour, il n'y a qu'une seule association féminine active dans le domaine de la production laitière et les femmes sont sous-représentées dans les organisations paysannes mixtes. Au niveau des instances dirigeantes, elles n'occupent pas des postes leur permettant de faire prévaloir leurs compétences. Au sein de la Maison des éleveurs, par exemple, une seule femme siège au sein d'un conseil d'administration de 15 personnes.

\section{Tableau III}

Revenus journaliers tirés de la vente de produits laitiers à Sédhiou : études de cas de deux femmes transformatrices de lait

\begin{tabular}{|c|c|c|c|c|c|c|c|c|c|}
\hline & \multicolumn{3}{|c|}{ Achat lait frais } & \multirow{2}{*}{$\begin{array}{l}\text { Autoconsom- } \\
\text { mation (I) }\end{array}$} & \multicolumn{3}{|c|}{ Vente lait caillé } & \multicolumn{2}{|c|}{ Revenus financiers (Fcfa) } \\
\hline & $\begin{array}{l}\text { Volume } \\
\text { (I) }\end{array}$ & $\begin{array}{c}\text { Prix } \\
(\text { Fcfa/l) }\end{array}$ & $\begin{array}{l}\text { Total } \\
\text { (Fcfa) }\end{array}$ & & $\begin{array}{l}\text { Volume } \\
\text { (I) }\end{array}$ & $\begin{array}{c}\text { Prix } \\
\text { (Fcfa/l) }\end{array}$ & $\begin{array}{l}\text { Total } \\
\text { (Fcfa) }\end{array}$ & $\begin{array}{l}\text { Journalier } \\
\text { (lait caillé) }\end{array}$ & $\begin{array}{c}\text { Saisonnier } \\
\text { (huile de beurre) }\end{array}$ \\
\hline \multicolumn{10}{|c|}{ Femme 1} \\
\hline SP & 25 & 150 & 3750 & 2 & 23 & 250 & 5750 & 2000 & 23500 \\
\hline SS & 15 & 175 & 2625 & 1 & 14 & 300 & 4200 & 1575 & \\
\hline \multicolumn{10}{|c|}{ Femme 2} \\
\hline SP & 35 & 175 & 6125 & 3 & 32 & 250 & 8000 & 1875 & 44800 \\
\hline SS & 15 & 175 & 2625 & 1 & 14 & 300 & 4200 & 1575 & \\
\hline
\end{tabular}

* Les revenus de la vente d'huile de beurre peuvent augmenter si les techniques de transformation sont améliorées. Actuellement, il faut 20 litres de lait pour un litre d'huile. NB : le lait est livré à domicile dans les deux cas et les coûts de transformation sont nuls (pas de ferments, ni de pasteurisation...). La vente se fait également à domicile. La femme 2 est obligée d'avoir un prix d'achat fixe toute l'année, condition pour une livraison en saison sèche

$\mathrm{SP}=$ saison des pluies $; \mathrm{SS}=$ saison sèche

\section{Tableau IV}

Implication des femmes dans les organisations paysannes de la région de Kolda

\begin{tabular}{|c|c|c|c|c|c|}
\hline Organisation & $\begin{array}{l}\text { Aire d'intervention/ } \\
\text { organisme d'appui }\end{array}$ & $\begin{array}{l}\text { Date de } \\
\text { création }\end{array}$ & Objectifs & $\begin{array}{l}\text { Total membres } \\
\text { (\% de femmes) }\end{array}$ & $\begin{array}{l}\text { Nb. membres } \\
\text { bureau ou } \\
\text { comité } \\
\text { (\% de femmes) }\end{array}$ \\
\hline Maison des éleveurs (Mde) & $\begin{array}{l}\text { Région Kolda/ } \\
\text { Direction de l'Elevage }\end{array}$ & 1996 & $\begin{array}{l}\text { Cadre de concertation } \\
\text { pour acteurs } \\
\text { des filières animales }\end{array}$ & 6000 (n.d.) & $15(6,6)$ \\
\hline $\begin{array}{l}\text { Directoire des femmes } \\
\text { en élevage (Dfe) }\end{array}$ & $\begin{array}{l}\text { Région Kolda/ } \\
\text { Direction de l'Elevage }\end{array}$ & 1996 & $\begin{array}{l}\text { Information, formation } \\
\text { de la femme rurale }\end{array}$ & $240(100)$ & $8(100)$ \\
\hline $\begin{array}{l}\text { Fédération communautaire } \\
\text { des agropasteurs de Sansamba } \\
\text { (Fecaps) }\end{array}$ & $\begin{array}{l}\text { Département Sédhiou/ } \\
\text { Primoca }\end{array}$ & 1990 & $\begin{array}{l}\text { Appui technique et } \\
\text { matériel des producteurs }\end{array}$ & $1682(0)$ & $7(0)$ \\
\hline $\begin{array}{l}\text { Association des jeunes } \\
\text { agropasteurs du département } \\
\text { de Sédhiou (Ajapds) }\end{array}$ & $\begin{array}{l}\text { Département Sédhiou/ } \\
\text { Primoca }\end{array}$ & 1996 & $\begin{array}{l}\text { Transformation et } \\
\text { commercialisation de lait }\end{array}$ & $6(0)$ & $6(0)$ \\
\hline $\begin{array}{l}\text { Groupement d'intérêt } \\
\text { économique (informel) (Tessito) }\end{array}$ & $\begin{array}{l}\text { Département Kolda/ } \\
\text { Sodefitex }\end{array}$ & 1998 & $\begin{array}{l}\text { Transformation et } \\
\text { commercialisation de lait }\end{array}$ & $122(100)$ & $6(100)$ \\
\hline $\begin{array}{l}\text { Union des éleveurs laitiers } \\
\text { de Bantancounto (UELB) }\end{array}$ & $\begin{array}{l}\text { Département Kolda/ } \\
\text { Vsf }\end{array}$ & 1998 & $\begin{array}{l}\text { Acquisition intrants } \\
\text { commercialisation de lait }\end{array}$ & n.d. (0) & n.d. (0) \\
\hline $\begin{array}{l}\text { Pinnal Fouladou: } \\
\text { union de } 12 \text { groupements }\end{array}$ & $\begin{array}{l}\text { Département Vélingara/ } \\
\text { FONGS }\end{array}$ & 1997 & $\begin{array}{l}\text { Transformation et } \\
\text { commercialisation de lait }\end{array}$ & $500(40)$ & $4(75)$ \\
\hline
\end{tabular}

n.d. : non défini

Primoca : Projet intégré pour la Moyenne Casamance ; Sodefitex :Société de développement des fibres textiles ; Vsf : Vétérinaires sans frontière ; FONGS : Fédération des organisations non-gouvernementales du Sénégal 
Les organisations paysannes les moins fonctionnelles sont celles mises en place au niveau régional par la puissance publique. Les objectifs visés et les actions à entreprendre ne sont pas toujours clairs, même pour les dirigeants. Elles ont chacune un projet général d'ordre social ou économique souvent mobilisateur, mais elles ont des difficultés à le traduire en actions concrètes. Certains producteurs se sont posés des questions sur leur utilité, d'autant plus qu'ils ont cotisé régulièrement et que le bénéfice matériel n'a pas encore été clairement identifié.

Les auteurs ont noté des écarts importants de compétence entre les leaders et les adhérents de base. La tendance à la monopolisation de l'information et au cumul des responsabilités par quelques personnes nuit au fonctionnement démocratique des organisations. Ceci est particulièrement vrai au sein des organisations féminines. Les femmes leaders sont en majorité des responsables politiques vivant en ville.

Des insuffisances de gestion technique et financière (management, gestion de la qualité, commercialisation...) ont été à l'origine de dysfonctionnements dans certaines associations. Les activités génératrices de revenus ne sont soutenues par aucune documentation comptable fiable, ce qui exclut toute possibilité de contrôle ou de maîtrise des flux financiers. L'absence de résultats concrets significatifs a tendance à démobiliser les membres.

\section{DISCUSSION}

La filière lait à Kolda est caractérisée par la prédominance du circuit traditionnel de transformation et de commercialisation des produits. Cette situation, commune à l'ensemble des régions du Sénégal (5), pose des problèmes de qualité des produits mis en marché. Pour garantir la qualité et la sécurité des produits offerts aux consommateurs, une amélioration durable des techniques de traite et de transformation doit être envisagée en s'appuyant sur des actions de formation. Les thèmes sont l'hygiène de la traite, les opérations primaires pour garder la qualité du lait et éviter son acidification rapide (pasteurisation), le contrôle des fermentations, les techniques de barattage manuel et de cuisson utilisées pour la fabrication de l'huile de beurre. L'utilisation de petit matériel facilement utilisable et lavable (écrémeuse à main, bidons et pots en aluminium...) devrait être encouragée.

La division du travail dans la filière lait dans la région de Kolda repose avant tout sur le sexe et l'âge. Le rôle essentiel et traditionnel de l'homme était de s'occuper uniquement du pastorat. Jusqu'à une époque récente, l'homme ne s'est jamais intéressé à la transformation, ni à la commercialisation du lait local $(2,12)$. Son intervention en la matière remonterait à 1994, suite à la naissance des premières ceintures laitières (7). Elle pourrait signifier une volonté d'accaparement d'un créneau naguère féminin, assurant des revenus assez réguliers. Dans les sociétés rurales, les hommes cherchent toujours à monopoliser la vente des produits des femmes et à contrôler leurs revenus (1). La création des unités de pasteurisation gérées par des hommes relève, en revanche, de la volonté des organismes d'appui de lutter contre le chômage des jeunes citadins.

La pratique de traite par les femmes est une pratique traditionnelle chez les Peuls (2). Elle peut être un interdit dans d'autres communautés comme chez les Touaregs du Niger, où la traite est exclusivement une activité masculine (18). L'importance de la part des femmes dans le troupeau bovin familial Peul a été déjà rapportée par d'autres auteurs $(2,4,12)$ qui ont également souligné l'absence de droit légal des femmes à disposer librement de leurs animaux. Le chef de famille peut se passer de l'accord de ses enfants ou de ses épouses avant de vendre leurs animaux (12). La prise de décision, au sein de la famille Peule, est une prérogative masculine parmi d'autres, telles qu'elles sont définies au sein de normes socioculturelles et à l'instar de nombreuses sociétés rurales des pays en développement (14).
Les produits laitiers ont représenté des revenus assez substantiels. Ils sont la base même de l'économie quotidienne du ménage Peul qui, par la vente de lait caillé et d'huile de beurre, subvient théoriquement à ses besoins alimentaires surtout en saison des pluies (d'après un dicton : «pas de beurre, pas de mil ») (11).

Peu de femmes ont reçu dans la région une formation technique sur la transformation du lait. Ces formations, très limitées dans le temps et dans l'espace, n'ont pas d'ailleurs produit les effets escomptés, en raison de l'analphabétisme de certaines femmes. Le manque de support pédagogique facilite l'oubli des enseignements reçus et ne milite pas en faveur d'une application rigoureuse des connaissances. Or, l'analphabétisme est chronique dans cette région de Kolda où 90 p. 100 des ruraux ne savent ni lire, ni écrire $(3,10)$. L'alphabétisation fonctionnelle paraît donc extrêmement utile pour maîtriser dans un langage accessible les nouvelles techniques de production ou de transformation. L'alphabétisation pourrait également faciliter la compréhension et l'accès aux mécanismes et principes de gestion et de comptabilité.

Le manque de temps a souvent été évoqué par les hommes pour justifier l'absence de femmes lors des séances de formation aux techniques de production ou lors des séances de discussions. Les femmes manquent de temps disponible à cause de la lourdeur des tâches ménagères (16). Des mesures d'accompagnement, comme l'acquisition de matériels tendant à alléger le travail des femmes, sont donc à promouvoir (foyers améliorés, moulins à mil, presses à huile, charrette pour le transport...). Ces petites réalisations font partie des conditions de réussite des programmes de développement agricole dans lesquels les femmes sont impliquées (17). L'allègement du travail domestique leur permet de disposer davantage de temps pour garantir leur participation aux séances de formation et d'animation.

Les organisations professionnelles présentes ont des difficultés de fonctionnement. Elles peinent à traduire en actions concrètes leurs projets, en l'absence non seulement de compétences en matière de planification et de programmation, mais aussi de formation adéquate et parfois d'appuis appropriés. Mais le développement d'organisations professionnelles fortes, capables de se prendre en charge, est également en partie freiné par "l'organisation sociale héritée de la tradition et certaines caractéristiques socioculturelles » (15), ainsi que par « un discours paternaliste des autorités et touffu de promesses mirobolantes, qui a fini de conforter les ruraux dans la conviction et la croyance qu'ils doivent tout attendre de l'Etat » (6). Or, face au désengagement croissant de l'Etat, les producteurs ne peuvent participer aux prises de décision que s'ils se dotent de structures à la fois représentatives et mandatées par eux. Cela suppose la prise de conscience de leurs forces et la volonté de renforcement de leurs capacités.

\section{- CONCLUSION}

Les femmes jouent un rôle important dans la filière laitière dans la zone périurbaine de Kolda. Elles sont propriétaires d'animaux, font la traite et assurent la transformation et la commercialisation du lait. Elles devraient donc être associées à tout processus de développement de cette filière dans la région. Des modules spécifiques de formation aux technologies de production et de transformation, à l'organisation d'associations de productrices, à la commercialisation, en particulier à l'approche commerciale commune pour les groupements de producteurs, aux notions de comptabilité et de gestion de microactivités pourraient aider à leur meilleure insertion dans la filière. Le renforcement des capacités à long terme devrait directement contribuer à l'implication croissante des femmes dans les associations paysannes et dans le processus d'élaboration des politiques locales. 


\section{Remerciements}

Les auteurs remercient l'ensemble des producteurs de la région de Kolda, les représentants des organisations paysannes, les responsables des projets de développement et des systèmes décentralisés de crédit pour leur disponibilité. La contribution de Cheikh Omar Ba de l'Isra a été très appréciée pour ses commentaires et suggestions. Nous le remercions vivement, ainsi qu'Eh Fallou Guèye de l'Isra et Philippe Lhoste du Cirad pour la lecture critique du document.

\section{BIBLIOGRAPHIE}

1. AKELLO G., SARR F., 1999. Le développement économique des femmes dans le développement agricole et rural : promouvoir les activités génératrices de revenus. Rapport annuel 1999 : en point de mire. Wageningen, Pays-Bas, Cta, $10 \mathrm{p}$.

2. BA C., 1986. Les Peul du Sénégal : étude géographique. Dakar, Sénégal, Nouvelles éditions africaines, $394 \mathrm{p}$.

3. BLANKEN J., BA C.O., BROETZ G., DIOP M., 2001. Etude portant sur les potentialités de développement en milieu rural. Dakar, Sénégal, Gtz, $235 \mathrm{p}$.

4. BONHOURE A.C., GAUTHIER L., 1997. Etudes comparées des pratiques agricoles dans trois villages du département de Kolda en Haute Casamance (Sénégal). Etude des déterminants de la gestion de la fertilité. Tome I. Montpellier, France, Cnearc-Esat, $176 \mathrm{p}$.

5. BROUTIN C., DIOKHANE O., 2000. La filière « lait et produits laitiers" au Sénégal. Dakar, Sénégal, Gret, Tpa, 38 p.

6. CISSOKHO M., 1994. La perception paysanne du développement agricole durable : le métier de paysan dans un environnement écologique, politique et économique difficile. In : Benoit-Cattin M., De Grandi J.-C. éds, Promotion de systèmes agricoles durables dans les pays d'Afrique soudano-sahélienne. Rome, Italie, FAO, p. 199-203.

7. COLLEUILLE A., DIEYE P.N., MBODJ A., 1999. La filière laitière en Haute Casamance et au Sénégal oriental. In : Actes de l'atelier de réflexion Isra-Crz Kolda/Sodefitex/Vsf, Kolda, 8-9 avril 1999. Kolda, Sénégal, Vsf, $15 \mathrm{p}$.

\section{Summary}

Ba Diao M., Senghor C.D., Diao B. Women in the Suburban Subsector of Milk Production and Processing in Senegal. Case of Kolda Area

In 2000, a participatory survey was carried out in Kolda area, in the Sudanese zone of Southern Senegal, to analyze constraints to development of the milk subsector and to determine women's role and contribution to this subsector. Women are often cattle legal owners. They also hand milk cows, and process and market dairy products. However, they have no control over means of production; it is difficult for them to have access to education and credit, and they little partake in farmers' organizations. To improve their status, it will be necessary to set up a development program that will include training and basic literacy, as well as support measures, e.g. equipment availability to reduce housework, and basic infrastructure development.

Key words: Role of women - Milk production - Participation of women - Educational policy - Farmers' association Senegal.
8. CONWAY G.R., MCCRACKEN J., PRETTY J., 1988. Introduction au diagnostic rapide pour le développement en milieu rural. Londres, Royaume-Uni, IIED, 24 p.

9. DIEYE P.N., 1997. Etude de la production laitière de la vache Ndama dans les systèmes de production mixtes de la zone subhumide du Sénégal. Mémoire de Confirmation, Isra, Kolda, Sénégal, 48 p.

10. DIREL, 1998. Programme de développement de l'élevage au Sénégal oriental et en Haute Casamance. Dakar, Sénégal, Direction de I'Elevage, $32 \mathrm{p}$.

11. DUPIRE M., 1962. Les facteurs humains de l'économie pastorale. Niamey, Niger, Ifan-Cnrs, 61 p. (Etudes nigériennes 6)

12. DUPIRE M., 1970. Organisation sociale des Peuls. Etude d'ethnographie comparée. Thèse Doct. es-Lettres, université Paris, France, Plon, 624 p.

13. FAO, 1994. Women, agriculture and rural development: a synthesis report of the Africa region. Rome, Italy, FAO, $58 \mathrm{p}$.

14. KOOPMAN J., 1997. Parité entre hommes et femmes et participation à la planification du développement agricole. Dix études de cas : ce qu'il faut en retenir. Service des femmes dans le développement. Rome, Italie, $\mathrm{FAO}, 63 \mathrm{p}$

15. MERCOIRET M.-R., 1994. Participation populaire et durabilité. In : Benoit-Cattin M., De Grandi J.-C. éds., Promotion de systèmes agricoles durables dans les pays d'Afrique soudano-sahélienne. Rome, Italie, FAO, p. 185-197.

16. MONIMART R., 1989. Femmes du Sahel : la désertification au quotidien. Paris, France, Karthala, Ocde, 154 p.

17. NDIAYE O.K., 1997. Parité entre hommes et femmes et participation à la planification du développement agricole. Leçons du Sénégal. Service des femmes dans le développement. Dakar, Sénégal, FAO, 36 p.

18. OXBY C., 1990. Peuples pasteurs en crise : les réponses des ONG en Afrique. Paris, France, Syros-Alternatives, $144 \mathrm{p}$.

19. SOW F., 1998. Quand I'une n'est pas I'autre : à propos des rôles sociaux des sexes. Afr. Dev., $33: 5-12$.

20. Technologie Développement Consultance, 2000. Les opportunités d'affaires dans la filière laitière de la région de Kolda. Dakar, Sénégal, Dynaentreprises/USAid, 95 p.

Reçu le 07.02.2002, accepté le 14.11.2003

\section{Resumen}

Ba Diao M., Senghor C.D., Diao B. Las mujeres en la filial lechera peri-urbana en Senegal. El caso de la región de Kolda

En el año 2000, se realizó una encuesta participativa en la región de Kolda, zona sudanesa al sur de Senegal, con el fin de analizar los obstáculos al desarrollo de la filial lechera y determinar el papel y el aporte de las mujeres en esta filial. Las mujeres son a menudo las propietarias jurídicas de los bovinos, se encargan del ordeño y aseguran la transformación y comercialización de la leche. Sin embargo, no posen ningún control sobre los factores de producción, el acceso a la instrucción y al crédito les es difícil y su nivel de participación en las organizaciones campesinas es bajo. La mejora de su situación pasa necesariamente por el establecimiento de un programa de apoyo, que comprenda acciones de formación y de alfabetización funcional, así como medidas de acompañamiento, tales como proporcionar materiales que faciliten los trabajos domésticos y la construcción de infraestructuras físicas de base.

Palabras clave: Papel de la mujer - Producción lechera - Participación de la mujer - Política educacional - Associación de agricultores - Senegal. 